\title{
A SIMPLE GENERATION METHOD OF PHYSICAL RANDOM DIGITS
}

\author{
Shunsuke Kishimoto*
}

\begin{abstract}
A simple method of generating physical random digits is described. In this method, an electronic decade counter is incremented by the clock pulses. As the overflow is ignored, the counter keeps counting from 0 to 9 . Whenever a micro-computer is interrupted by a rare random signal caused by a radiant ray detection, a digit in the counter is latched and loaded in the memory. These digits are used as the random numbers of one figure or several figures. This method is the most simple one of the traditional methods. A mathematical estimation for the occurrence probabilities of each digit suggests that the maximum deviation from the equiprobabilities $1 / 10$, is less than $\left(6 \times 10^{-9}\right)$.
\end{abstract}

\section{Introduction}

To obtain an ideal random number sequence, past authors have tried physical generations with the aid of natural random events. Ishida and Ikeda(1956) utilized the radioactivity of Cobalt 60 as a noise source. Jansson(1966) reported technique based on electron noise in valves and radiation from radioactive substances. Miyatake et al.(1975) and Miyatake et al.(1979) also used Cobalt 60 and Cesium 137 as gamma-ray sources.

Kishimoto et al.(1981) proposed a new physical method using the radioactivity of Cesium 137 as a random source. In this method, the physical device consists of a radiation detector, a small scale micro-computer and some electronic circuits. The process of random digit generation is as follows;

(1) A decade counter counts the number of $1 \mathrm{MHz}$ clock pulses from the quartz oscillator, and repeats the counting from 0 to 9 .

(2) When a gamma-ray emitted from Cesium 137 is detected, a content of the counter, one of the digits of $0,1, \ldots, 9$, is latched by a buffer circuit used as a high speed register.

(3) A micro-computer reads the register and saves the digit on a floppy disk. Then it resets the counter to zero.

*Tsuyama National College of Technology, Tsuyama, Okayama, 708, Japan.

Key words: Random number; Physical random number; Physical random digit; Random digit generation. 


\section{KISHIMOTO}

Under this condition of which the counting rate of gamma-rays is about $100 \mathrm{~Hz},\left(8 \times 10^{6}\right)$ random digits are generated and recorded on floppy diskettes. The maximum deviation from the occurrence probability of $1 / 10$ for each digit was estimated at less than $\left(5 \times 10^{-5}\right)$.

Taking a few of the features of the method for instance: (a) It is the most simple one of those attempted in the past. (b) It generates the random decimal numbers directly. (c) We can easily get the mathematical estimation for the occurrence probabilities of each digit.

In this paper, we are going to improve the generation method by decreasing the deviation without spoiling the simplicity. We will also estimate the maximum value of the deviation in the reformed method.

\section{Physical device}

The schematic diagram of generation is shown on Fig.1. The quartz oscillator generates clock pulses of a constant time-interval. The pulses are counted by a synchronous up/down decade counter. The content of the counter repeats from 0 to 9 over and over again. It is transferred to the buffer register when a strobe pulse arrives.

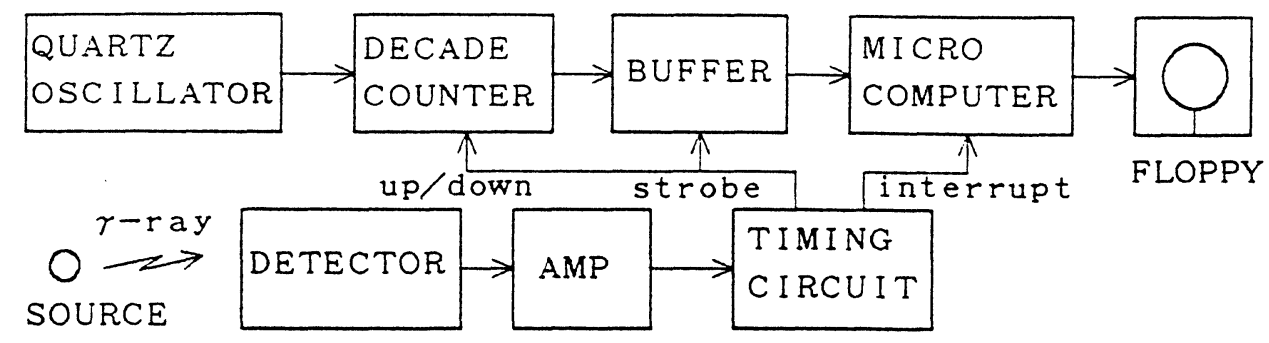

Figure 1: Outline of random digit generation

On the other hand, the gamma-ray pulses put out by the detector are amplified and formed into the standard logic level. We call it $\gamma$-pulse. The $\gamma$-pulse can be utilized as a random strobe pulse. So, whenever $\gamma$-pulse occurs, a digit in the decade counter at that instant is latched in the buffer register. The $\gamma$-pulse is also used as an interrupt request signal to make the micro-computer read the buffer. The micro-computer reads the digit from the buffer and stores it in the memory. Since the operation of the counter and the gamma-ray detection are independent events, the digits read by the micro-computer must have a certain randomness.

This generation process is the same as past attempts. We are going to try some improvements of the generation method so as to decrease the deviation of the equiprobabilities. In the old generation method, the decade counter counted the number of clock pulses only in the up-count state, and was then reset to zero just after the strobe pulse arrived. On the other hand, in the new generation method, it is reset to zero and set in up-count operation, or preset to 9 and set in down-count operation alternately just after the strobe pulse occurs. We reformed the electronic circuit by using a synchronous up/down decade counter with 


\section{A Simple Generation Method of Physical Random Digits}

4-bit programmable data inputs. The inputs can load any 4-bit preset data. The loading is synchronous with the clock pulses.

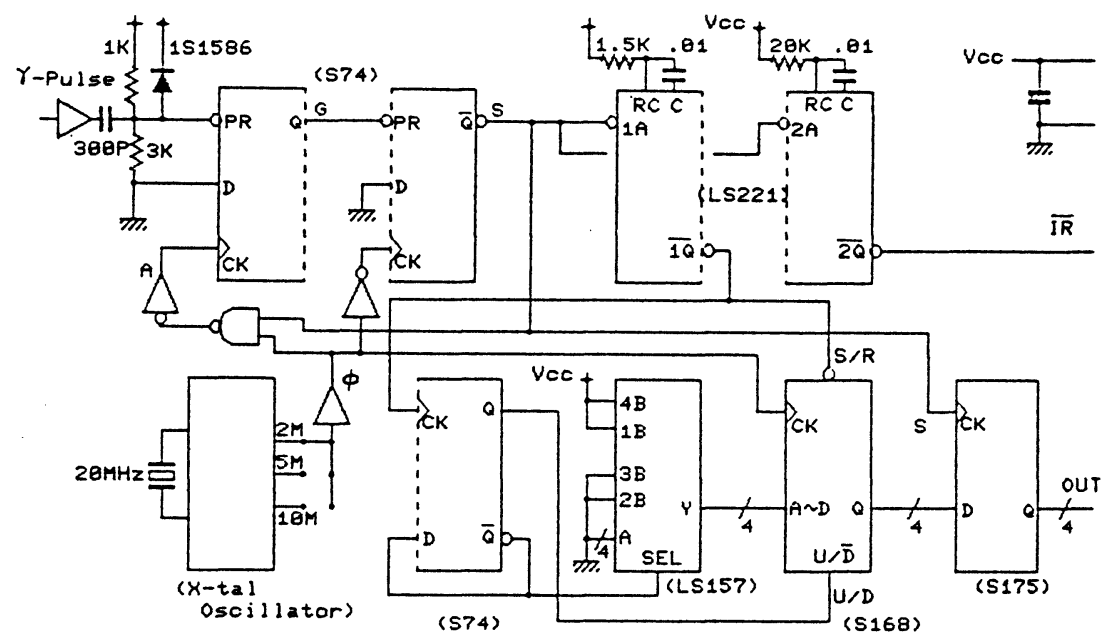

Figure 2: A circuit of random digit generator

Other improvements are to increase the clock frequency up to $2 \sim 10 \mathrm{MHz}$, and the usage of high speed type logic IC for the part which requests quick response. The remade electronic circuit generating the random digit sequence is shown in Fig.2 and the timing chart of the circuit is shown in Fig.3.

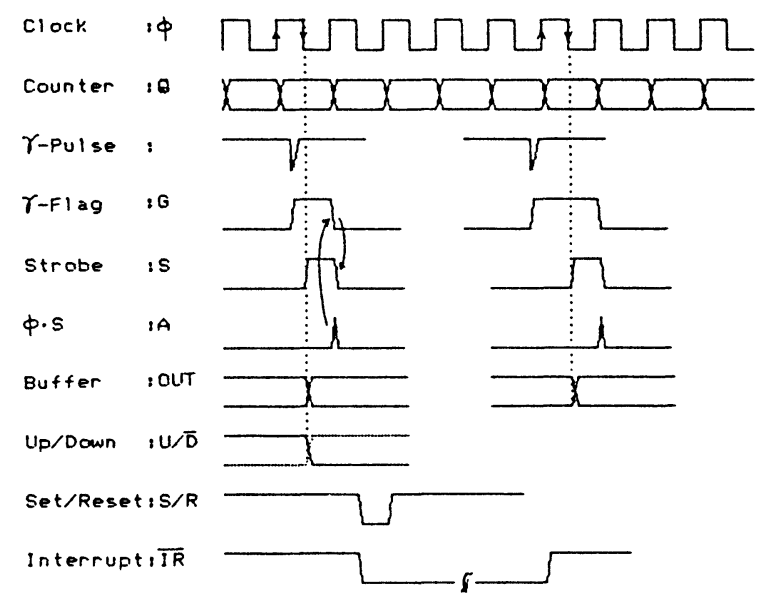

Figure 3: Signal timing chart of the generator

In Fig.3, $\gamma$-flag signal is set at a high level when $\gamma$-pulse occurs, and then reset to a low level by the leading edge of the clock pulse just after the strobe pulse is set at a high level. It is used as a flag to avoid reading an unstable state of the counter when $\gamma$-pulse coincides with a transition time of the counter. 


\section{KISHIMOTO}

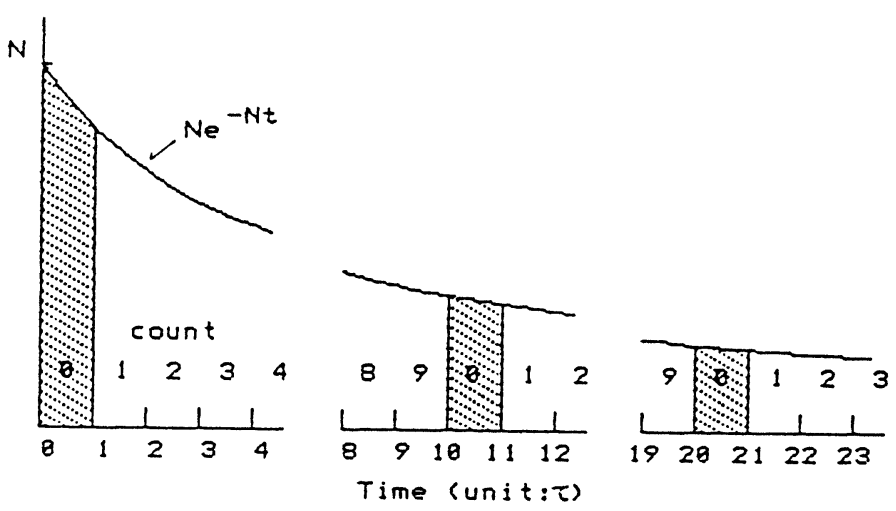

Figure 4: Probability density curve of the radioactive decay phenomena

The counting rate of gamma-rays is set easily by adjusting the distance between the source and the detector. The frequency of the clock pulses can be selected from one of $2 \mathrm{MHz}, 5 \mathrm{MHz}$ or $10 \mathrm{MHz}$.

\section{Generation probability of each digit}

As the half life of Cesium 137 is about 30 years, the probability that a nucleus of Cesium will decay in the unit time is about $\left(7 \times 10^{-10}\right)$. The probability is small enough and the decay phenomena occur independently. So the frequency that gamma-ray pulses caused by the numerous nuclear disintegration have the typical Poisson distribution. Let $N$ is the average frequency per second. Then $1-e^{-N t}$ is the probability that the gamma-ray is detected at least once by the time $t$ and $N e^{-N t}$ is the probability density function.

Under the condition that the counter is in the up-count state, we can get the probability of the digit 0 by calculating the shadowed area in Fig.4.

Then the probability $P u(i)$ of getting the digit $i(i=0,1, \ldots, 9)$ is given by

$$
P u(i)=\sum_{n=0}^{\infty} \int_{(10 n+i) \tau}^{(10 n+i+1) \tau} N e^{-N t} d t=\frac{\left(1-e^{-N \tau}\right) e^{-N i \tau}}{1-e^{-10 N \tau}}
$$

where $\tau$ is the period of the clock pulses. In the case of $(N \tau \ll 1)$, this is approximated by

$$
P u(i) \doteq \frac{1}{10}\left(1-\frac{2 i-9}{2} N \tau+\frac{i^{2}-9 i+12}{2} N^{2} \tau^{2}\right)
$$

When the decade counter is in the down-count state, the probability $P d(i)$ is calculated by replacing $i$ with $9-i$.

$$
P d(i) \doteq \frac{1}{10}\left(1+\frac{2 i-9}{2} N \tau+\frac{i^{2}-9 i+12}{2} N^{2} \tau^{2}\right) .
$$


As we operate the counter in the up-count state and in the down-count state alternately, the total probability $P(i)$ of getting the digit $i$ is

$$
P(i) \doteq \frac{1}{2} P u(i)+\frac{1}{2} P d(i)=\frac{1}{10}\left(1+\frac{i^{2}-9 i+12}{2} N^{2} \tau^{2}\right) .
$$

In our generation condition, $N=200 \mathrm{~Hz}, \tau=0.5 \mu \mathrm{sec}$, and $N \tau=10^{-4} \cdot(M a x)\left(i^{2}-9 i+\right.$ $12) / 20 \mid=0.6$.) As a result, the maximum deviation from the equiprobabilities $1 / 10$, is

$$
\varepsilon_{m}=|P(i)-1 / 10|<6 \times 10^{-9} \text {. }
$$

This value is sufficiently small in comparision with the past attempts.

\section{Results and Conclusion}

About $\left(2 \times 10^{7}\right)$ random digits are generated and recorded on floppy diskettes under the condition that the counting rate of the gamma-rays is $200 \mathrm{~Hz}$ and the frequency of the clock pulse is $2 \mathrm{MHz}$.

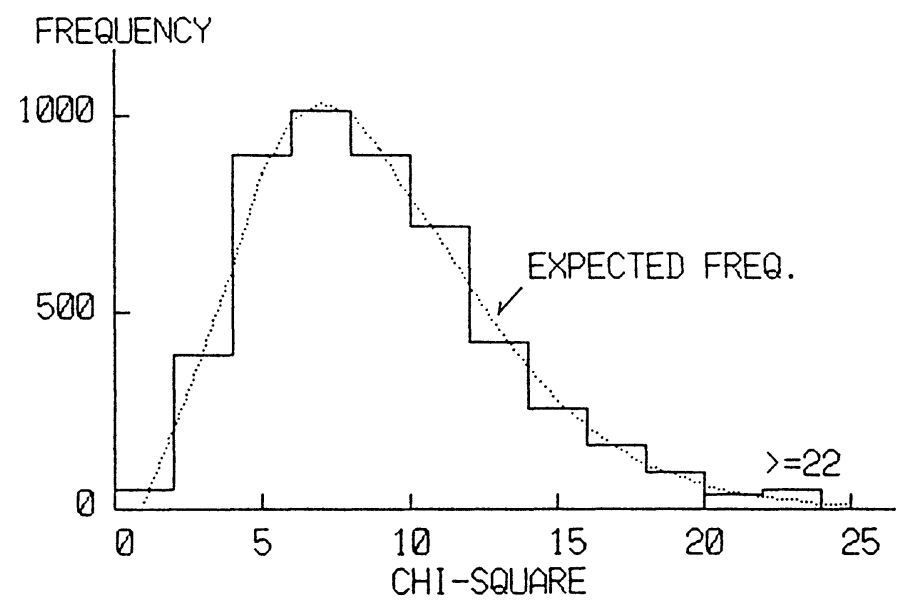

Figure 5: A histogram of $\chi^{2}$-values of the frequency test

The frequency tests have been applied to the random digits. That is, all digits are divided into 5000 blocks, 4000 digits each. The value of $\left(\chi_{9}^{2}\right)$ for each block is evaluated by the formula $\sum_{i=0}^{9} \frac{\left(n_{i}-400\right)^{2}}{400}$, where $\left(n_{i}\right)$ is the observed number of digit $i$.

The frequency histogram of $\chi^{2}$-values for 5000 blocks is shown in Fig.5. Here the value of the $\chi^{2}$ has been divided into 12 equal classes. Also examine whether the distribution of these $5000 \chi^{2}$ 's is fitted to the $\chi^{2}$ distribution with the degree of freedom 11 or not. The dotted curve in Fig. 5 is the expected frequency of $\chi^{2}$-distribution, where the degree of freedom is 


\section{KISHIMOTO}

11. The result is $\left(\chi^{2}=10.51\right)$. Let $\chi_{11}^{2}$ represent the random variable of the $\chi^{2}$-distribution with the degree of freedom 11 , then $\left(P\left[\chi_{11}^{2} \geq 10.34\right]=0.50\right)$ and $\left(P\left[\chi_{11}^{2} \geq 12.90\right]=0.30\right)$. We can find the upper side probability of $\left(\chi^{2}=10.51\right)$ between $50 \%$ and $30 \%$.

To check on the degree of independence of each digit, we calculate the serial correlation coefficient of lag $k$.

$$
r_{k}=\frac{\frac{1}{n} \sum_{i=1}^{n} d_{i} d_{i+k}-\bar{d}^{2}}{\frac{1}{n} \sum_{i=1}^{n}\left(d_{i}-\bar{d}\right)^{2}}
$$

where $d_{i}$ is the $i$ th digit of our random sequence and $\left(\bar{d}=\frac{1}{n} \sum_{i=1}^{n} d_{i}\right)$. Fig.6 shows the results in case of $k=1$ and 3. When $n$ is greater than $\left(1 \times 10^{6}\right)$, the coefficient $r_{k}$ becomes less than $\left( \pm 2 \times 10^{-3}\right)$ in both cases.

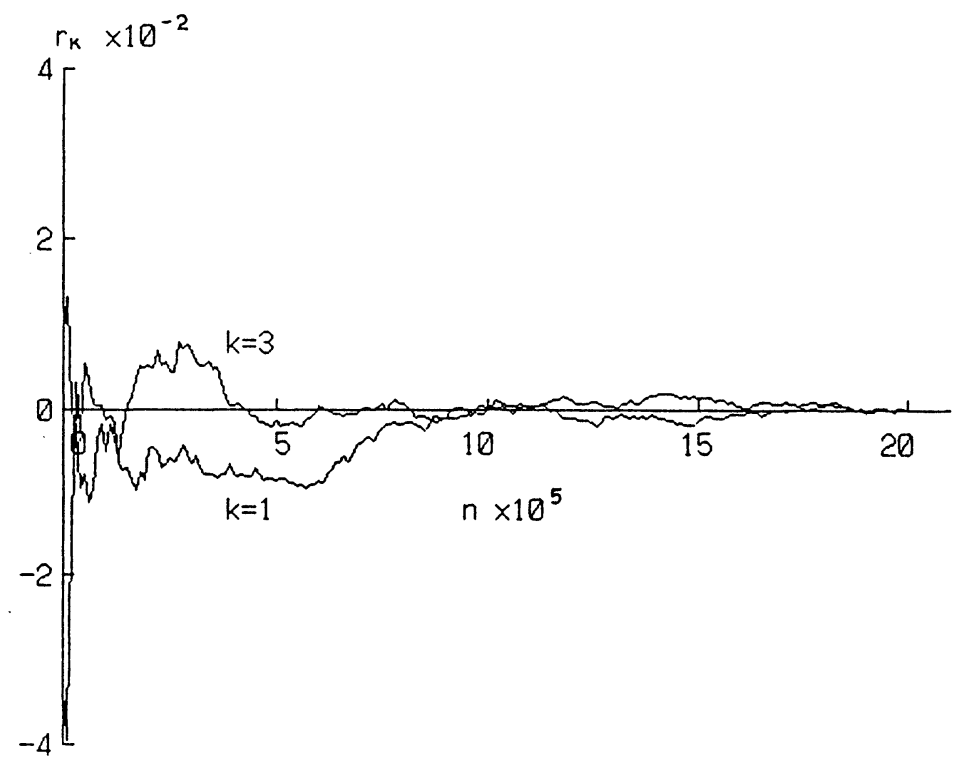

Figure 6: Serial correlation test

The results of these two tests, it seems that the random numbers generated by our method have good quality.

As the electronic circuits of our random digit generator are small, all of them are built in a universal board that can be inserted in the auxiliary slot of the micro-computer. Therefore, the apparatus is easily movable and maintenance-free. If we adjust the detection rate of radiation to a very slow speed, we can see that the random digits are projected one by one on the CRT screen of the micro-computer in real time. Therefore, we will use our apparatus as a teaching aid to study some statistical distributions and real time simulations. 


\section{A Simple Generation Method of Physical Random Digits}

\section{Acknowledgement}

The author thanks Prof. T. Midoro and Prof. T. Sugiyama of Tsuyama National College of Technorogy for valuable discussions. The author wishes to thank Prof. E. Ohtaki of Okayama University for his useful suggestions.

\section{REFERENCES}

Ishida,M. and Tkeda,H.(1956). Random number generator, Ann. Inst. Statist. Math., 8, 119-126.

Jansson,B.(1966). Random Number Generators, Victor Pettersons Bokindustri Aktiebolag, Stockholm, 283-305.

Kishimoto,S., Ikegami,T. and Hiramatsu,M.(1981). A generation method of physical random number sequence by using micro-computer, J.Japan Statist. Soc., 11, 2, 169-173.

Miyatake,O., Inoue,H. and Yoshizawa,Y.(1975). Generation of physical random numbers, Math. Japon., 20, 207-217.

Miyatake,O., Yoshizawa,Y., Inoue,H., Kumahora,H., Ichimura,M.

and Kimura,H.(1979). On the generation and properties of physical random numbers, Math. Japon., 20, 369-376.

(Received March 1993; Revised October 1993) 